\title{
Author Correction: A comprehensive model for assessment of liver stage therapies targeting Plasmodium vivax and Plasmodium falciparum
}

\author{
Alison Roth (1) 1, Steven P. Maher (10 1,2, Amy J. Conway', Ratawan Ubalee ${ }^{3}$, Victor Chaumeau 4,5, \\ Chiara Andolina ${ }^{4,5}$, Stephen A. Kaba ${ }^{6}$, Amélie Vantaux ${ }^{7}$, Malina A. Bakowski ${ }^{8}$, Richard Thomson-Luque ${ }^{1}$ \\ Swamy Rakesh Adapa ${ }^{1}$, Naresh Singh', Samantha J. Barnes' ${ }^{1}$, Caitlin A. Cooper ${ }^{2}$, Mélanie Rouillier ${ }^{9}$, \\ Case W. McNamara ${ }^{8}$, Sebastian A. Mikolajczak ${ }^{10}$, Noah Sather ${ }^{10}$, Benoit Witkowski ${ }^{8}$, Brice Campo ${ }^{9}$, \\ Stefan H.I. Kappe ${ }^{10}$, David E. Lanar ${ }^{6}$, François Nosten (1) 4,5, Silas Davidson ${ }^{3}$, Rays H.Y. Jiang (1) ${ }^{1}$, \\ Dennis E. Kyle (iD ${ }^{1,2} \&$ John H. Adams (iD) ${ }^{1}$
}

Correction to: Nature Communications https://doi.org/10.1038/s41467-018-04221-9, published online 09 May 2018.

The original version of this article contained an error in the spelling of Richard Thomson-Luque, which was incorrectly given as Richard Thomson Luque. This error has now been corrected in both the PDF and HTML versions of the Article.

Published online: 08 June 2018

\begin{abstract}
(c) Open Access This article is licensed under a Creative Commons Attribution 4.0 International License, which permits use, sharing, adaptation, distribution and By reproduction in any medium or format, as long as you give appropriate credit to the original author(s) and the source, provide a link to the Creative Commons license, and indicate if changes were made. The images or other third party material in this article are included in the article's Creative Commons license, unless indicated otherwise in a credit line to the material. If material is not included in the article's Creative Commons license and your intended use is not permitted by statutory regulation or exceeds the permitted use, you will need to obtain permission directly from the copyright holder. To view a copy of this license, visit http://creativecommons.org/licenses/by/4.0/.
\end{abstract}

(C) The Author(s) 2018

\footnotetext{
${ }^{1}$ Department of Global Health, College of Public Health, Center for Global Health and Infectious Diseases Research, University of South Florida, 3720 Spectrum Blvd 404, 33612 Tampa, FL, USA. ${ }^{2}$ Center for Tropical and Emerging Global Diseases, University of Georgia, 500 DW Brooks Dr. Suite 370,30602 Athens, GA, USA. ${ }^{3}$ Department of Entomology, Armed Forces Research Institute of Medical Sciences (AFRIMS), 315/6 Rajvithi Rd, 10400 Bangkok, Thailand. ${ }^{4}$ Centre for Tropical Medicine and Global Health, Nuffield Department of Medicine, University of Oxford, Oxford, UK. ${ }^{5}$ Shoklo Malaria Research Unit, Mahidol Oxford Research Unit, Faculty of Tropical Medicine, Mahidol University, 68/30 Bantung Rd, 63110 Mae Sot, Tak, Thailand. ${ }^{6}$ Malaria Vaccine Branch, Walter Reed Army Institute of Research, 503 Robert Grant Ave, 20910 Silver Spring, MD, USA. ${ }^{7}$ Malaria Molecular Epidemiology Unit, Institut Pasteur du Cambodge, 5 Boulevard Monivong-PO Box 983, 12201 Phnom Penh, Cambodia. ${ }^{8}$ California Institute for Biomedical Research (Calibr), 11119N Torrey Pines Rd, Suite 100, 92037 La Jolla, CA, USA. ${ }^{9}$ Medicines for Malaria Venture, Pré-Bois Rd 20, 1215 Meyrin, Switzerland. ${ }^{10}$ Center for Infectious Disease Research, 307 Westlake Ave N Suite 500, 98109 Seattle, WA, USA. Correspondence and requests for materials should be addressed to J.H.A. (email: jadams3@health.usf.edu)
} 